\title{
A UNIQUENESS CONDITION FOR SEQUENCES
}

\author{
STEVEN LETH
}

\begin{abstract}
Under certain conditions a sequence of real numbers converging to zero is determined up to a constant multiple by the comparisons of its subsums. One such condition is that each number in the sequence be majorized by the sum of the elements beyond it.
\end{abstract}

A uniqueness condition for sequences. A sequence $\left\langle a_{n}\right\rangle$ of real numbers induces an ordering on subsets of $N$ in the following way:

$$
I \leqslant J \quad \text { iff } \quad \sum_{i \in I} a_{i} \leqslant \sum_{j \in J} a_{j} .
$$

We ask the question: Under what conditions does the induced ordering determine the sequence (up to a constant multiple)? A partial answer to this question is given by the theorem below, as well as by the remarks and counterexamples at the end of the paper.

I would like to thank the referee for his extremely helpful suggestions. He also points out that Proposition 1 below is essentially an old Putnam problem (1955A3).

Definitions. We say that $\left\langle a_{n}\right\rangle$ and $\left\langle b_{n}\right\rangle$ are two sympathetic sequences, and write $\left\langle a_{n}\right\rangle \sim\left\langle b_{n}\right\rangle$ iff $\left\langle a_{n}\right\rangle$ and $\left\langle b_{n}\right\rangle$ induce the same ordering on subsets of $N$. We note that if $\left\langle a_{n}\right\rangle \sim\left\langle b_{n}\right\rangle$, then $\sum_{i \in I} a_{i}=\sum_{j \in J} a_{j}$ iff $\sum_{i \in I} b_{i}=\sum_{j \in J} b_{j}$. Also, if $\left.a_{n}\right\rangle$ $\sum_{k=n+1}^{\infty} a_{k}$ we say that $a_{n}$ is a bully. The existence of bullies in a sequence is strongly related to the original question. In particular we have the following.

THEOREM. Let $\left\langle a_{n}\right\rangle$ and $\left\langle b_{n}\right\rangle$ be two nonincreasing sequences of real numbers with no bullies such that $\left.a_{n}>0, b_{n}\right\rangle 0, a_{n} \rightarrow 0, b_{n} \rightarrow 0$ and $\left\langle a_{n}\right\rangle \sim\left\langle b_{n}\right\rangle$. Then there is an $\alpha$ such that $a_{n}=\alpha b_{n}$ for all $n$.

COROLLARY. If, in addition, $\sum_{n=1}^{\infty} a_{n}=\sum_{n=1}^{\infty} b_{n}=1$, then $a_{n}=b_{n}$ for all $n$.

The corollary answers a question of Chauqui and Malitz. It states that a probability measure on a $\sigma$-complete atomic Boolean algebra is unique if it respects a preordering (a total, transitive, complete extension of the Boolean ordering) which includes the condition $a_{n} \leqslant \sum_{k=n+1}^{\infty} a_{k}$ for all $n$ (no bullies in the Boolean ordering). Chuaqui and Malitz establish the existence of a compatible measure in this case assuming certain natural conditions on the preordering [CM]. Their conditions are a (nearly complete) characterization of which orderings on $2^{N}$ are induced by sequences.

Received by the editors May 31, 1983 and, in revised form, February 8, 1984.

1980 Mathematics Subject Classification. Primary 40B05, 40A10, $26 \mathrm{D} 15$.

(C)1985 American Mathematical Society $0002-9939 / 85 \$ 1.00+\$ .25$ per page 
Proposition 1. Suppose $\left\langle u_{n}\right\rangle$ is a nonincreasing sequence with $u_{n} \rightarrow 0$ and no bullies and let $v$ be any real number such that $0 \leqslant v \leqslant \sum_{k=1}^{\infty} u_{k}$. Then there exists an infinite subset $I \subseteq N$ such that $v=\sum_{I} u_{i}$. (Note. If $\sum u_{n}$ diverges, then there are no bullies and $v$ may be any nonnegative number.)

Proof. Define $I$ inductively by the Greedy Algorithm. If $v>u$, then $1 \in I$; once $I_{n}(=I \cap\{1, \ldots, n\})$ has been determined, $n+1 \in I_{n+1}$ iff

$$
\sum_{I_{n}} u_{i}=i_{n+1}<v \text {. }
$$

Define $v_{n}=\sum_{I_{n}} u_{i}$. We note that $v>v_{n} \geqslant v_{n-1}$, so that $v_{n} \uparrow v_{0} \leqslant v$. If $v_{0}=v$, we are done. Otherwise, let $d=v-v_{0}>0$. From (*), if $r \notin I$, then $v_{r-1}+u_{r}>v$ so that $u_{r}>d$. Thus only finitely many $r$ are not in $I$. If no indices are omitted, then $I=N$ and $\sum_{k=1}^{\infty} u_{k}<v$, a contradiction. Otherwise let $s$ be the last omitted index. Then

$$
v_{s-1}+u_{s} \geqslant x>v_{s-1}+\sum_{k=s+1}^{\infty} u_{k}
$$

so that $u_{s}$ is a bully, a contradiction.

Proposition 2. For $j$ fixed, let $M_{j}=\left\{i: b_{i} / a_{i} \neq b_{j} / a_{j}\right\}$. If $\left\langle a_{n}\right\rangle \sim\left\langle b_{n}\right\rangle$, then $\Sigma_{M_{j}} a_{k}$ and $\Sigma_{M_{j}} b_{k}$ are both finite.

COROLlARY. If $\left\langle a_{n}\right\rangle \sim\left\langle b_{n}\right\rangle$ and $\left\langle b_{n}\right\rangle$ is not a multiple of $\left\langle a_{n}\right\rangle$, then $\sum a_{i}<\infty$ and $\sum b_{i}<\infty$.

Proof. Suppose $b_{r} \neq b_{1} a_{r} / a_{1}$ for some $r$. Since $N=M_{1} \cup M_{r}, \sum a_{i} \leqslant \sum_{M_{1}} a_{i}+$ $\sum_{M_{r}} a_{i}<\infty$. Similarly, $\sum b_{i}<\infty$.

Proof OF Proposition 2. Let $k_{r}=b_{r} / a_{r}, M_{j}^{+}=\left\{i: k_{i}>k_{j}\right\}$ and $M_{j}^{-}=\{i$ : $\left.k_{i}<k_{j}\right\}$. Since $M_{j}=M_{j}^{+} \cup M_{j}^{-}$and the situation is symmetric, it suffices to prove that $\sum_{M_{j}^{+}} a_{i}$ and $\Sigma_{M_{j}^{+}} b_{i}$ are both finite. Suppose not; then $b_{i}>k_{j} a_{i}$ on $M_{j}^{+}$implies that $\sum_{M_{j}^{+}} b_{i}$ diverges if either diverges. Choose $s>j$ so that $s \in M_{j}^{+}, a_{j}>a_{s}$ and $b_{j}>b_{s}$ and write $b_{s}=k_{j} a_{s}+\varepsilon$. Let $x=b_{j}-b_{s}+\varepsilon / 2$. We assume (for contradiction) that $\Sigma_{M_{j}^{+}} b_{i}$ diverges, so that Proposition 1 can now be applied to $\left\langle b_{n}\right\rangle$ restricted to $M_{j}^{+}-\{s\}$. Choose $J_{0} \subset M_{j}^{+}-\{s\}$ such that $\Sigma_{J_{0}} b_{i}=x$ and let $J=J_{0}$ $\subset\{s\}$. Then $\sum_{J} b_{i}=b_{s}+x=b_{j}+\varepsilon / 2>b_{j}$. On the other hand,

$$
\begin{aligned}
\sum_{J} a_{i} & =a_{s}+\sum_{J_{0}} a_{i} \leqslant a_{s}+k_{j}^{-1} \sum_{J_{0}} b_{i}=a_{s}+k_{j}^{-1}\left(b_{j}-b_{r}+\varepsilon / 2\right) \\
& =k_{j}^{-1}\left(b_{r}-\varepsilon+b_{j}-b_{r}+\varepsilon / 2\right)=k_{j}^{-1}\left(b_{j}-\varepsilon / 2\right)<a_{j} .
\end{aligned}
$$

This violates the sympathy of $\left\langle a_{n}\right\rangle$ and $\left\langle b_{n}\right\rangle$.

LEMMA. If $\left\langle a_{n}\right\rangle \sim\left\langle b_{n}\right\rangle$ and $\sum a_{n}=\sum b_{n}$, then there are infinitely many $n$ such that $a_{n} \leqslant b_{n}$, and infinitely many $n$ such that $a_{n} \geqslant b_{n}$.

Proof. Suppose not, and obtain an $N$ such that (w.l.o.g.), for all $k>N, a_{k}>b_{k}$. By Proposition 1 we may write $a_{N}=\sum_{I} a_{i}$, where $I$ is infinite (so that $\min I>N$ ), and since $\left\langle a_{n}\right\rangle \sim\left\langle b_{n}\right\rangle b_{N}=\sum_{I} b_{i}$, so that $a_{N}>b_{N}$. Thus (by backward induction), $a_{n}>b_{n}$ for all $n$, and so $\sum a_{n}>\sum b_{n}$, a contradiction. 
Proof of The Theorem. By the Corollary to Proposition 2 it suffices to prove the corollary to the Theorem. Define

$$
f(x)=\sum_{I_{x}} b_{i}, \quad \text { where } I_{x} \text { is such that } x=\sum_{I_{x}} a_{i} .
$$

Note that such an $I_{x}$ always exists for any $x \in[0,1]$ by Proposition 1 . Since $\langle a\rangle \sim\langle b\rangle$, it is clear that $f$ is well defined and that if $x \leqslant y$, then $f(x) \leqslant f(y)$. Thus $f$ is a monotone function taking $[0,1]$ into $[0,1]$, with $f(0)=0$ and $f(1)=1$.

By Lebesgue's theorem on monotone functions, this implies that $f^{\prime}(x)$ exists a.e., and that $\int_{a}^{b} f^{\prime}(x) d x \leqslant f(b)-f(a)$ for any $[a, b] \subseteq[0,1]$ (see, for example, [R, p. 96]). Now pick any $x$ such that $f^{\prime}(x)$ exists, and obtain $I_{x}$ as before. Define

$$
h_{n}= \begin{cases}a_{n} & \text { if } n \notin I_{x}, \\ -a_{n} & \text { if } n \in I_{x} .\end{cases}
$$

Then if $a_{n} \notin I_{x}$,

$$
\frac{f\left(x+h_{n}\right)-f(x)}{h_{n}}=\frac{\sum_{I_{x}} b_{i}+b_{n}-\sum_{I_{x}} b_{i}}{a_{n}}=\frac{b_{n}}{a_{n}},
$$

while $a_{n} \in I_{x}$ implies

$$
\frac{f\left(x+h_{n}\right)-f(x)}{h_{n}}=\frac{\sum_{I_{x}} b_{i}-b_{n}-\sum_{I_{x}} b_{i}}{-a_{n}}=\frac{b_{n}}{a_{n}} .
$$

We now have $\lim _{n \rightarrow \infty}\left(b_{n} / a_{n}\right)$ exists, and so by the $\operatorname{Lemma~}_{n \rightarrow \infty}\left(b_{n} / a_{n}\right)=1$, and thus $f^{\prime}(x)=1$ a.e.

Finally, suppose that there is a point $c$ such that $f(c) \neq c$. If $f(c)>c$, then

$$
\int_{c}^{1} f^{\prime}(x) d x=1-c>f(1)-f(c) \text {, }
$$

while $f(c)<c$ yields

$$
\int_{0}^{c} f^{\prime}(x) d x=c-0>f(c)-f(0) .
$$

Since either situation is impossible, it must be that $f(x)=x$ on $[0,1]$, and that, in particular, $a_{n}=b_{n}$ for all $n$.

REMARK. It is evident that the condition "no bullies" is necessary in order to have every $x \in\left[0, \sum a_{n}\right]$ expressible as a sum of $a_{i}$ 's, for if $a_{n}>\sum_{k=n+1}^{\infty} a_{k}$, then any $x$ between the two cannot possibly be so written.

Necessary and sufficient conditions for the conclusion of the Theorem are more elusive. The following examples will establish: (1) The Theorem is not true if the condition "no bullies" is removed. (2) It is possible that the ordering induced by $\left\langle a_{n}\right\rangle$ determines the sequence up to a constant multiple but that $\left\langle a_{n}\right\rangle$ has no bullies.

EXAMPLE 1. Any two sequences $\left\langle a_{n}\right\rangle$ and $\left\langle b_{n}\right\rangle$ in which every element is a bully are clearly sympathetic. Thus, for example, the sequences $\left\langle a r^{n}\right\rangle$ with $0<r<\frac{1}{2}$ are all sympathetic. More generally, call two finite sequences $\left\{d_{1}, \ldots, d_{r}\right\}$ and $\left\{e_{1}, \ldots, e_{r}\right\}$ $r$-sympathetic if, for $I, J \subset\{1, \ldots, r\}, \Sigma_{I} d_{i} \geqslant \Sigma_{J} d_{j}$ iff $\sum_{I} e_{i} \geqslant \sum_{J} e_{j}$. It is evident that there are many sets of $r$-sympathetic sequences which are not proportional. Now 
construct sequences $\left\langle a_{n}\right\rangle$ and $\left\langle b_{n}\right\rangle$ out of blocks of $r(i)$-sympathetic strings, $r(i) \geqslant 1$ :

$$
\begin{aligned}
& \langle a\rangle=\left\{d_{1}^{1}, \ldots, d_{r(1)}^{1}, d^{2}, \ldots, d_{r(2)}^{2}, \ldots\right\}, \\
& \langle b\rangle=\left\{e^{1}, \ldots, e_{r(1)}^{1}, e^{2}, \ldots, e_{r(2)}^{2}, \ldots\right\}
\end{aligned}
$$

subject to the conditions

$$
\sum_{I} d_{i}^{k}-\sum_{J} d_{j}^{k}>\sum_{n=k+1}^{\infty} \sum_{m=1}^{r(n)} d_{m}^{n} \text { and } \sum_{I} e_{i}^{k}-\sum_{J} e_{j}^{k}>\sum_{n=k+1}^{\infty} \sum_{m=1}^{r(n)} e_{m}^{n}
$$

whenever the left sides are positive and $I, J \subset\{1, \ldots, r(k)\}$.

Now, the ordering on $2^{N}$ induced by $\left\langle a_{n}\right\rangle$ is given by:

$I=J$ iff the order induced within each block is equal.

$I>J$ iff the first block in which the induced order is unequal gives $I_{k}>J_{k}$, where $I_{k}$ and $J_{k}$ are the elements of $I$ and $J$ within the $k$ th block. By construction and the block-by-block sympathy of $\left\langle a_{n}\right\rangle$ and $\left\langle b_{n}\right\rangle$ it is clear that $\left\langle b_{n}\right\rangle$ induces the same order so that $\left\langle a_{n}\right\rangle \sim\left\langle b_{n}\right\rangle$, although they need not be proportional.

EXAMPLE 2. Let $\left\langle a_{n}\right\rangle$ be the sequence whose sum is one determined by the following set of equalities:

$$
\begin{gathered}
a_{3 n+1}=a_{3 n+2}+\sum_{k=3 n+4}^{\infty} a_{k}, \quad a_{3 n+2}=\sum_{k=3 n+3}^{\infty} a_{k}, \\
a_{3 n+1}+\sum_{k=3 n+4}^{\infty} a_{k}=a_{3 n+2}+a_{3 n+3} .
\end{gathered}
$$

The inequalities for $n=0$ force $a_{1}=4 / 10, a_{2}=3 / 10, a_{3}=2 / 10$ and $\sum_{k=4}^{\infty} a_{k}=$ $1 / 10$ (so that $a_{3}>\sum_{k=4}^{\infty} a_{k}$ ). This can easily be seen by substituting $4 / 10+\varepsilon_{1}$, $3 / 10+\varepsilon_{2}, 2 / 10+\varepsilon_{3}$ and $1 / 10+\varepsilon_{4}$ for $a_{1}, a_{2}, a_{3}$ and $\sum_{k=4} a_{k}$, respectively, and checking that the only solution (given that $\sum a_{k}=1$ ) is $\varepsilon_{1}=\varepsilon_{2}=\varepsilon_{3}=\varepsilon_{4}=0$. In exactly the same way it can be checked that $a_{3 n+1}=4 / 10^{n+1}, a_{3 n+2}=3 / 10^{n+1}$, $a_{3 n+3}=2 / 10^{n+1}$ and $\sum_{k=3 n+4}^{\infty} a_{k}=1 / 10^{n+1}$, so that every $a_{3 n+3}$ is a bully, even though the induced order determines $\left\langle a_{n}\right\rangle$ uniquely.

Open questions. (1) What are necessary and sufficient conditions for the conclusion of the theorem? An answer to this question might be of use in anwering open questions in $[\mathbf{C M}]$ also.

In the absence of a complete answer to (1), the following might be interesting:

(2) Are all sympathetic sequences which are not proportional given by block bullies as in Example 1?

(3) In Example 2 we had uniqueness even though every third element was a bully. Could we have every other element a bully?

\section{REFERENCES}

[CM] R. Chuaqui and J. Malitz, Preorderings compatible with probability measures, Trans. Amer. Math. Soc. 279 (1983), 811-824.

[R] H. L. Royden, Real analysis, 2nd ed., Macmillan, New York, 1968.

Department of Mathematics, University of Colorado, Boulder, Colorado 80309 